\title{
Self-activated ultrahigh chemosensitivity of oxide thin film nanostructures for
}

SUBJECT AREAS:

ELECTRONIC MATERIALS

AND DEVICES

SENSORS

MATERIALS PHYSICS

APPLIED PHYSICS

Received

18 May 2012

Accepted

2 August 2012

Published

17 August 2012

\section{Correspondence and} requests for materials should be addressed to S.-J.Y. (sjyoon@kist.re. kr) or H.W.J.

(hwjang@snu.ac.kr) \section{transparent sensors}

\author{
Hi Gyu Moon 1,2, Young-Soek Shim' ', Do Hong Kim', Hu Young Jeong ${ }^{3}$, Myoungho Jeong ${ }^{4}$, \\ Joo Young Jung ${ }^{5}$, Seung Min Han ${ }^{5}$, Jong Kyu Kim ${ }^{6}$, Jin-Sang Kim ${ }^{1}$, Hyung-Ho Park ${ }^{2}$, Jong-Heun Lee ${ }^{7}$, \\ Harry L. Tuller ${ }^{8}$, Seok-Jin Yoon' \& Ho Won Jang ${ }^{9}$
}

\begin{abstract}
${ }^{1}$ Electronic Materials Research Center, Korea Institute of Science and Technology, Seoul 136-791, Republic of Korea, ${ }^{2}$ Department of Materials Science and Engineering, Yonsei University, Seoul 120-749, Republic of Korea, ${ }^{3}$ UNIST Central Research Facilities, Ulsan National Institute of Science and Technology, Ulsan 689-798, Republic of Korea, ${ }^{4}$ Department of Materials Science and Engineering, Korea Advanced Institute of Science and Technology, Daejeon 305-701, Republic of Korea, ${ }^{5}$ Graduate School of Energy Environment Water Sustainability, Korea Advanced Institute of Science and Technology, Daejeon 305-701, Republic of Korea, ${ }^{6}$ Department of Materials Science and Engineering, Pohang Institute of Science and Technology, Pohang 790-784, Republic of Korea, ${ }^{7}$ Department of Materials Science and Engineering, Korea University, Seoul 136-713, Republic of Korea, ${ }^{8}$ Department of Materials Science and Engineering, Massachusetts Institute of Technology, Cambridge, Massachusetts 02139, USA, ${ }^{9}$ Department of Materials Science and Engineering, Research Institute of Advanced Materials, Seoul National University, Seoul 151-744, Republic of Korea.
\end{abstract}

One of the top design priorities for semiconductor chemical sensors is developing simple, low-cost, sensitive and reliable sensors to be built in handheld devices. However, the need to implement heating elements in sensor devices, and the resulting high power consumption, remains a major obstacle for the realization of miniaturized and integrated chemoresistive thin film sensors based on metal oxides. Here we demonstrate structurally simple but extremely efficient all oxide chemoresistive sensors with $\sim 90 \%$ transmittance at visible wavelengths. Highly effective self-activation in anisotropically self-assembled nanocolumnar tungsten oxide thin films on glass substrate with indium-tin oxide electrodes enables ultrahigh response to nitrogen dioxide and volatile organic compounds with detection limits down to parts per trillion levels and power consumption less than $\mathbf{0 . 2}$ microwatts. Beyond the sensing performance, high transparency at visible wavelengths creates opportunities for their use in transparent electronic circuitry and optoelectronic devices with avenues for further functional convergence.

mart sensors, offering real-time analysis of gaseous chemical analytes, are essentials for environmental emissions monitoring, fossil fuel combustion control, medical diagnosis, artificial olfaction and homeland security ${ }^{1-9}$. Simplicity in operation, low cost, flexibility in production and small size constitute the main advantages of chemoresistive-type semiconductor chemical sensors based on metal oxides over electrochemical, optical, acoustic and other types of chemical sensors ${ }^{10-15}$. To insure rapid and reversible operation of such sensors, which rely on adsorption and desorption of molecules on the surface of semiconducting materials, the operation temperature is typically maintained between 200 and $400^{\circ} \mathrm{C}^{10,11}$. This requires the integration of heating elements within sensor devices with power consumption as high as hundreds of $\mathrm{mW}$. Micromachined silicon platforms reduce the power consumption down to tens of $\mathrm{mW}^{16}$, but this still remains a burden for portable devices operating with batteries. Ultralow power consumption of chemical sensors based on self-heated individual metal oxide nanowires ${ }^{17}$ or nanobelts ${ }^{18}$ have been reported, but these typically are insufficiently sensitive and/or are difficult to integrate with low-cost and high-yield mass production processes ${ }^{19}$. In addition to power consumption, high temperature operation of conventional semiconductor chemical sensors based on thin or thick films composed of metal oxide nanoparticles results in long-term instability, reflected in the drift of both sensor resistance and response ${ }^{9}$. Highly sensitive devices rely on the response of metal oxide nanoparticles within a thin or thick film matrix, which tend to aggregate and coarsen into larger secondary particles in order to lower their surface energies $^{20,21}$. The aggregation reduces film porosity and hampers the in-diffusion of chemical analytes. The coarsening leads to the decreased carrier modulation efficiency within the particles ${ }^{22}$. Although pre-aging 
thermal treatments at higher temperatures can improve long-term stability, the complete elimination of thermal degradation remains a problem, unless the operation temperature can be reduced below $200^{\circ} \mathrm{C}$, where grain growth normally initiates for nanoparticles of a few $\mathrm{nm}$ dimension ${ }^{23}$. At the same time, lowering the operation temperature below $200^{\circ} \mathrm{C}$ normally compromises sensitivity and response kinetics, as discussed above. Therefore, obtaining highly sensitive chemical sensors that operate at low temperatures $\left(<200^{\circ} \mathrm{C}\right)$ remains a challenging issue.

The key objective of this study is to achieve highly sensitive and stable chemoresistive metal oxide sensors with low power consumption by the nanostructuring of the sensing films which is designed for efficient self-activation. The response of sensing films to chemical analytes significantly increases as the feature sizes of the nanostructures approach the Debye length ${ }^{24-26}$. Using self-activation, the volume of the heated portion of the sensor can be minimized, while the heat dissipation to the substrate and ambient is dramatically reduced. Stable and extended operation is exploited by adjusting the working temperature of the sensing film via self-activation. Another key objective is to develop optically transparent high performance sensors with potential for being integrated into transparent electronics, automobile windshields and other transparent devices.
We believe such work would create a new paradigm for chemical sensors with opportunities in a wide range of here-to-fore untapped applications.

\section{Results}

The fabrication process of transparent chemical sensors in which commercially available indium-tin oxide (ITO)-coated glass was used as the substrate is shown in Fig. 1a. ITO interdigitated electrodes (IDEs) with interspacing of $5 \mu \mathrm{m}$ were patterned by dry etching. Porous $\mathrm{WO}_{3}$ thin films were deposited onto the IDEs by glancing angle deposition (GAD) via RF sputtering. GAD utilizes the selfshadowing effect of initial nuclei to grow inclined nanowires, nanorods or nanocolumns ${ }^{27-29}$. The nature of the oxide nanostructures grown by GAD depends largely on the material being sputtered ${ }^{27}$. For example, porous nanostructures were obtained for $\mathrm{WO}_{3}$ and $\mathrm{Nb}_{2} \mathrm{O}_{5}$, whereas $\mathrm{In}_{2} \mathrm{O}_{3}, \mathrm{TiO}_{2}, \mathrm{SnO}_{2}$ and $\mathrm{ZnO}$ formed relatively dense films under the same deposition condition (Supplementary Fig. S1). The size and density of the initial nuclei, which depend on the metal element, appear to be key parameters in determining the final nanostructure. Oxides composed of higher melting temperature metal elements result in more highly porous structures. Films with microstructural elements exhibiting high length to diameter aspect ratios
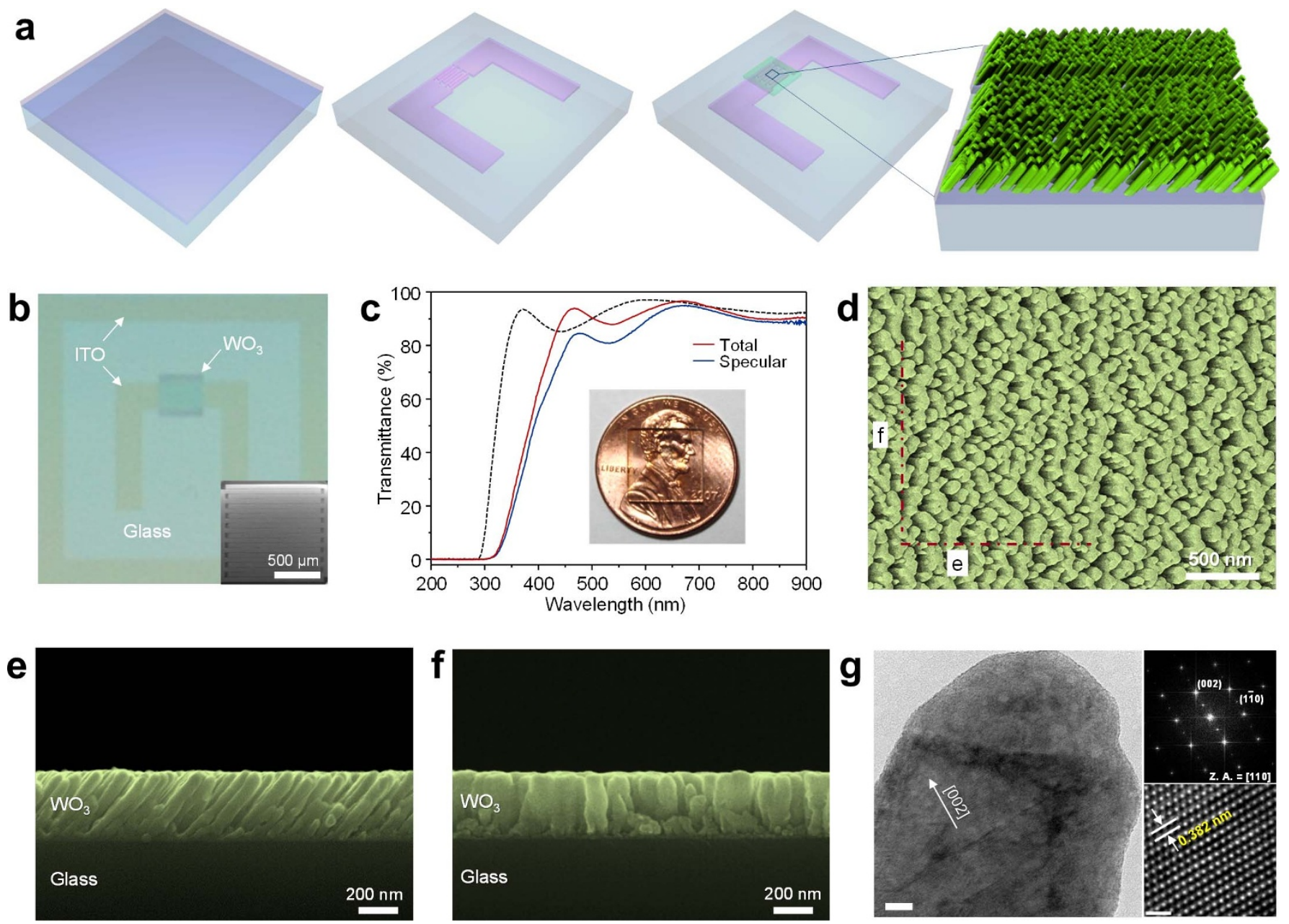

Figure 1 Transparent sensors based on nanocolumnar $\mathrm{WO}_{3}$ thin films. (a) Schematic showing the fabrication process of transparent sensors based on nanocolumnar oxide films. (b) Photograph of a fabricated sensor. Inset: SEM image of nanocolumnar $\mathrm{WO}_{3}$ film on ITO IDEs. (c) Total and specular transmittance spectra of the nanocolumnar $\mathrm{WO}_{3}$ film/ITO IDEs/glass sensor structure. For reference, the total transmittance spectrum of the ITO/glass substrate is presented (dash line). Inset: photograph of a sensor on a coin (penny). (d) Plain-view SEM image of the nanocolumnar $\mathrm{WO}_{3}$ film between ITO IDEs. (e), (f) Cross-sectional SEM images of the nanocolumnar $\mathrm{WO}_{3}$ film cut along (e) and across (f) the ITO IDEs as marked in (d). (g) Highresolution TEM image of $\mathrm{a} \mathrm{WO}_{3}$ nanocolumn. Scale bar, $10 \mathrm{~nm}$. Right: selected area diffraction pattern with zone axis of [110] (top) and lattice image of $\mathrm{WO}_{3}$ with interplanar spacing of $0.382 \mathrm{~nm}$ corresponding to the (002) plane (bottom). Scale bar, $1 \mathrm{~nm}$. 
are described as nanocolumnar. An example is the 330-nm-thick porous $\mathrm{WO}_{3}$ thin film, with column diameters of 30-80 nm and aspect ratios, ranging from 5 to 8 .

Figure $1 \mathrm{~b}$ shows a photograph of a fabricated sensor where the nanocolumnar $\mathrm{WO}_{3}$ sensing film on ITO IDEs looks smooth macroscopically. The total and specular transmittance spectra of the nanocolumnar $\mathrm{WO}_{3}$ film/ITO IDEs/glass sensor structure, measured from $200 \mathrm{~nm}$ to $900 \mathrm{~nm}$, are shown in Fig. 1c. The longer onset absorption wavelengths of the sensor relative to that of the ITO/glass substrate are due to the smaller optical band gap of $\mathrm{WO}_{3}$ $(3.0 \mathrm{eV})$ compared to that of ITO $(3.9 \mathrm{eV})^{30}$. After averaging the oscillations in the spectrum due to optical interference effects, the total transmittance of the sensor over $450-900 \mathrm{~nm}$ is determined to be $90.2 \%$, nearly identical to the transmittance of the ITO/glass substrate by itself. Owing to this high transmittance, the fabricated sensors are barely visible, as shown in the inset of Fig. 1c, suggesting that these sensors could be embedded in next-generation smart displays equipped with transparent electronics for automobile, biomedical, military, aerospace, and consumer applications ${ }^{31}$. With a maximum difference of $14 \%$ at $440 \mathrm{~nm}$, the $1.5 \%$ lower specular transmittance, on average, compared to the total transmittance means that light scattering likely takes place due to the presence of pores and local variations in the lengths of $\mathrm{WO}_{3}$ nanocolumns. Upon closer examination, it becomes apparent that the nanocolumnar $\mathrm{WO}_{3}$ films exhibit structural anisotropy (Fig. 1d). The crosssectional scanning electron microscopy (SEM) image of the films between the IDEs, cut parallel with the direction of the IDEs, show arrays of canted nanocolumns separated from each other by elongated pores which extend over nearly the full thickness of the film (Fig. 1e). In contrast, the nanocolumns are connected each other and form walls with considerably higher density across the IDEs (Fig. 1f). The pores facilitate access of gaseous molecules to the bottom of the columnar film, while the dense connected nanocolums across the IDEs provide efficient pathways for electrical current flow. The separation of the individual nanocolumns grown between the IDEs is seen more clearly by the cross-sectional transmission electron microscopy (TEM) images (Supplementary Fig. S2). High-resolution TEM (Fig. 1g) show that each nanocolumn has high crystallinity and (002) preferred orientation, even though the overall $\mathrm{WO}_{3}$ film with a monoclinic structure is polycrystalline as indicated by X-ray diffraction (Supplementary Fig. S2). By analysing the selected area diffraction patterns from TEM and X-ray
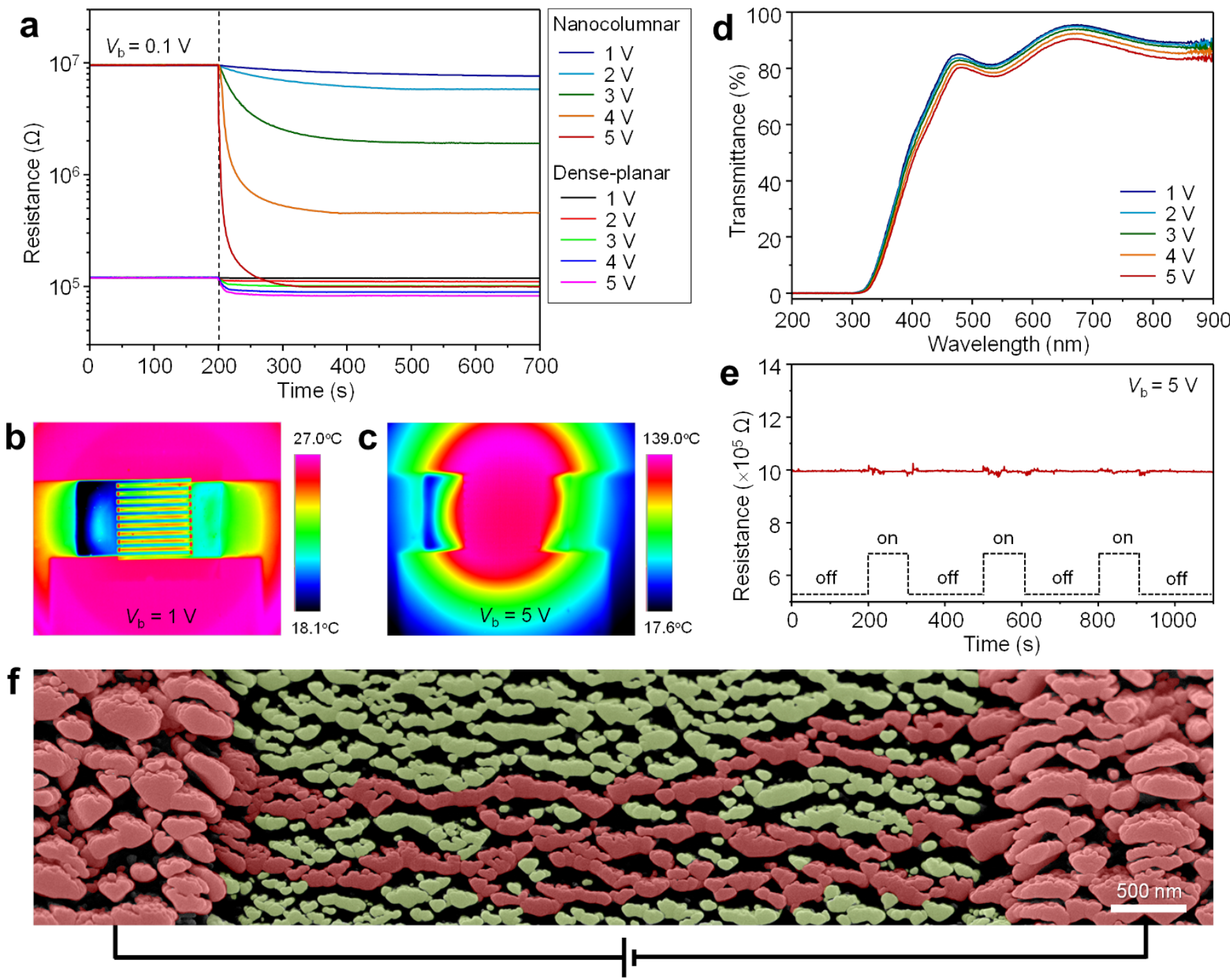

Figure $2 \mid$ Self-activation. (a) Resistance-time characteristics with variation of applied bias voltage for dense-planar and nanocolumnar $\mathrm{WO}_{3}$ thin film sensors. (b), (c) Thermographic images showing temperature variation in the $\mathrm{WO}_{3}$ thin film sensors with different bias voltages ( $V_{\mathrm{b}}$ ). (d) Bias -voltagedependent specular transmittance spectra of the nanocolumnar $\mathrm{WO}_{3}$ film/ITO IDEs/glass sensor structure. (e) Response of the nanocolumnar $\mathrm{WO}_{3}$ thin film sensor to office fluorescent light with a luminance of about $400 \mathrm{~lx}$ showing no change in device resistance. (f) $40^{\circ}$-tilted SEM image of nanocolumnar $\mathrm{WO}_{3}$ film between and on ITO IDEs. Parts highlighted in reddish color indicate localized current pathways which meander with narrow necks. 

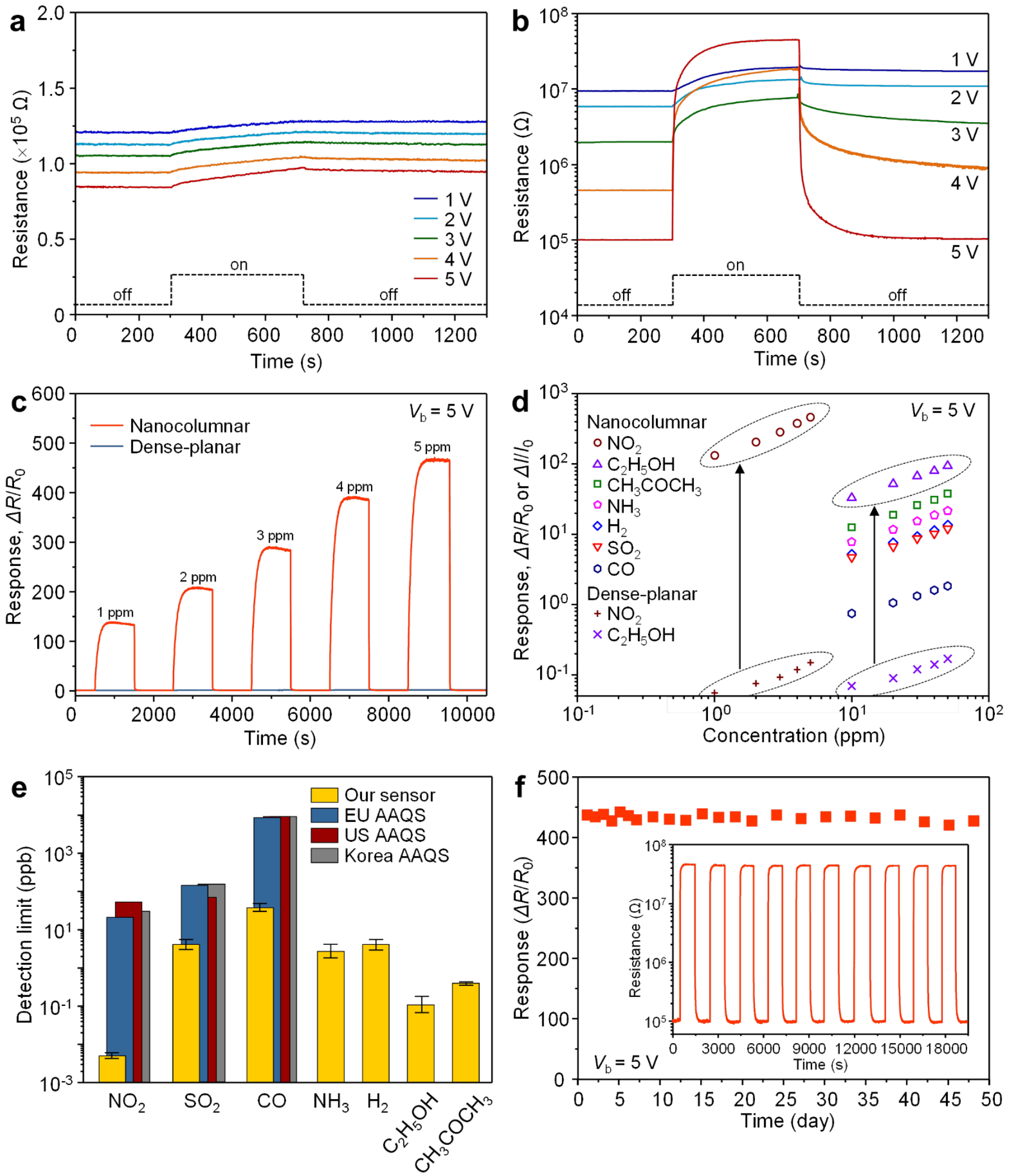

Figure 3 Gas sensing properties. (a), (b) Dynamic sensing transients of the dense-planar (a) and nanocolumnar $\mathrm{WO}_{3}$ thin film sensor (b) to 5 ppm $\mathrm{NO}_{2}$ with changing applied bias from 1 to $5 \mathrm{~V}$. Measurements were carried out without external heating. (c) Sensing transients of the dense-planar and nanocolumnar $\mathrm{WO}_{3}$ thin film sensors to $1-5 \mathrm{ppm} \mathrm{NO}_{2}$ at an applied bias voltage of $5 \mathrm{~V}$. (d) Response of the dense-planar and nanocolumnar $\mathrm{WO}_{3}$ thin film sensors as a function of gas concentration for various target gases. (e) Detection limits of the nanocolumnar $\mathrm{WO}_{3}$ thin film sensor to various gases. Ambient air quality standard (AAQS) levels of European Union (EU), United States (US), and Korea for $\mathrm{NO}_{2}, \mathrm{SO}_{2}$, and CO are presented for comparison. The averaging periods of AAQSs for $\mathrm{NO}_{2}, \mathrm{SO}_{2}$, and $\mathrm{CO}$ are 1 year, 1 hour, and 8 hours, respectively (see Supplementary Table $\mathrm{S} 1$ for details). (f) Change of response of nanocolumnar thin film sensor to $5 \mathrm{ppm} \mathrm{NO}_{2}$ for 48 days. Inset: response to 10 consecutive pulses of 5 ppm $\mathrm{NO}_{2}$ after 48 days of operation. 
diffraction patterns, it was revealed that the nancolumnar $\mathrm{WO}_{3}$ films are stoichiometric.

\section{Discussion}

Self-activation of dense-planar and nanocolumnar $\mathrm{WO}_{3}$ thin film sensors was monitored by measuring resistance change with increasing applied voltage. Both devices displayed linear current-voltage characteristics (Supplementary Fig. S3), indicating ohmic behaviour between the $\mathrm{WO}_{3}$ films and ITO electrodes ${ }^{30}$. When the applied voltage was increased from $0.1 \mathrm{~V}$ to $5 \mathrm{~V}$, there was a decrease by a factor of 1.45 in sensor resistance, with time transient of $\sim 150 \mathrm{~s}$, for the conventionally prepared thin film sensor (Fig. 2a). Thermographic images show that the increase in film temperature by self-activation was less than $2^{\circ} \mathrm{C}$ (Supplementary Fig. S4). For the same condition, the resistance of the nanocolumnar film decreased by two orders of magnitude with transient time of $\sim 120 \mathrm{~s}$. The film temperature rose to $139^{\circ} \mathrm{C}$ due to self-activation (Figs. 2b,c). The reduced resistance of the $\mathrm{WO}_{3}$ film at higher temperatures reflects the semiconducting nature of $\mathrm{WO}_{3}$. The nanocolumnar thin film device exhibits approximately two orders of magnitude higher resistance than the conventional film at the applied bias voltage of $0.1 \mathrm{~V}$, but due to the pronounced self-activation of the nanocolumnar film, both devices exhibit similar values of resistance under $5 \mathrm{~V}$ bias. Figure $2 \mathrm{~d}$ displays the bias voltage dependence of the specular transmittance spectra of the sensor. The small red shifts of the spectra with applied bias are consistent with a reduction in the bandgap energy of $\mathrm{WO}_{3}$ with temperature; additional evidence for voltage-induced selfactivation in the $\mathrm{WO}_{3}$ sensing film. The absence of response to surrounding light signals (Fig. 2e) confirms that the present sensor can be used without hindrance from ambient light.

Pronounced self-activation in the nanocolumnar film originates from the unusual geometry of the film. Figure $2 \mathrm{f}$ shows a $40^{\circ}$-tilted SEM image of the nanocolumnar $\mathrm{WO}_{3}$ film deposited between and on the ITO IDEs. The porosity of the film is estimated to be $38 \%$ on the basis of the analysis of the black and white contrast. A closer look reveals that the nanocolumns are disconnected from each other at many points and that some current pathways in the film are established only along localized regions. Even percolating pathways often meander through narrow necks $(20-40 \mathrm{~nm}$ in width). For this geometry, electron flow is constricted, leading to increased joule heating. Furthermore, ambient air in the pores, providing an excellent thermal barrier, suppresses heat dissipation laterally to the IDEs, while the small contact area between nanocolumns and glass substrate minimizes heat dissipation to the substrate. This is in contrast to large heat losses in conventional sensors, connected with the incorporation of independent heaters screen-printed or sputtered beneath the substrate or between the sensing film and the substrate. These findings clearly demonstrate that self-assembled nanocolumnar $\mathrm{WO}_{3}$ films serve as very efficient self-activated microheaters with minimal heat loss and power consumption. In addition to thermographic imaging, joule heating in the nanocolumnar films was confirmed by application of nanoscale electrical contact resistance (NanoECR) measurements (Supplementary Fig. S5).

Figures $3 \mathrm{a}, \mathrm{b}$ show the dynamic sensing transients of the denseplanar and nanocolumnar $\mathrm{WO}_{3}$ thin film sensors to $5 \mathrm{ppm} \mathrm{NO}_{2}$ without external heating. In the case of the dense-planar sensor, the response to $5 \mathrm{ppm} \mathrm{NO}$ (defined here as $\Delta R / R_{0}$, where $R_{0}$ and $\Delta R$ denote the initial resistance of the sensor in air and the resistance change of the sensor by exposure to the test gas, respectively) increased with increasing bias voltage (Fig. 3a). However, the response is as low as 0.15 at $5 \mathrm{~V}$ and the sensor does not show full recovery to the original resistance. In contrast, the nanocolumnar film sensor shows a dramatic enhancement in response with increase in bias voltage. At $5 \mathrm{~V}$, the response is extremely high, $\sim 450$; the highest value ever reported, as far as we are aware, for metal oxide thin film sensors ${ }^{11}$. The resistance fully recovers to the initial value within several hundred seconds. The response time $\left(t_{90}\right)$ that is the time for the sensor's response resistance to reach $90 \%$ of its steadystate value for the nanocolumnar film sensor is about $190 \mathrm{~s}$, which is much faster than that of the dense-planar sensor $\left(t_{90}>500 \mathrm{~s}\right)$. The linear and ultra-high response of the nanocolumnar sensor to 1-5 ppm of $\mathrm{NO}_{2}$ promises reliable detection of this environmentally important gas (Fig. 3c). In comparison, the response of the denseplanar sensor is negligibly small and sluggish, largely, attributable to the low sensing temperature and its reduced active surface area.

To verify the superior response of the nanocolumnar sensor relative to the dense-planar sensor more generally, the response to various gases such as $\mathrm{NO}_{2}, \mathrm{C}_{2} \mathrm{H}_{5} \mathrm{OH}$ (ethanol), $\mathrm{CH}_{3} \mathrm{COCH}_{3}$ (acetone), $\mathrm{NH}_{3}, \mathrm{H}_{2}, \mathrm{SO}_{2}$ and $\mathrm{CO}$ was studied. For the dense-planar sensor, the responses of the sensor to acetone, $\mathrm{NH}_{3}, \mathrm{H}_{2}, \mathrm{SO}_{2}$, and $\mathrm{CO}\left(\Delta I / I_{0}\right.$, where $I_{0}$ and $\Delta I$ denote the initial current of the sensor in air at a fixed bias and the current change by exposure to the test gas, respectively) were close to zero while the responses to $5 \mathrm{ppm} \mathrm{NO}_{2}$ and $50 \mathrm{ppm}$ ethanol were lower than 0.2 (Fig. $3 \mathrm{~d}$ ). In stark contrast, ultra-high responses to all the gases were achieved by the nanocolumnar sensor (Supplementary Fig. S6). For $\mathrm{NO}_{2}$ and ethanol, the response was three orders of magnitude higher than for that of the dense-planar sensor. This exceptional response of the nanocolumnar sensor is attributed to the combined effects of self-heating, the porous nanostructure with high surface-to-volume ratio $(\sim 33$ times higher specific area than the dense-planar film) and the presence of narrow necks between the columns (Supplementary Fig. S7). Although the $\mathrm{NO}_{2}$ concentration of $1 \mathrm{ppm}$ was the lowest examined experimentally in the present study, the theoretical detection limit ${ }^{32,33}$ (signal-tonoise ratio $>5$ ) was calculated to be approximately 5 parts per trillion (ppt) (Fig. 3e). This value is much lower than the ambient air quality standard (AAQS) levels of the European Union, United States and Korea, which are at several ppb levels (Supplementary Table S1). For $\mathrm{SO}_{2}$ and $\mathrm{CO}$, the detection limits of the nanocolumnar sensor are also substantially lower than the AAQS levels, suggesting strong potential of this technology serving as the basis of highly responsive air quality sensors. Furthermore, detection limits of sub-ppb levels to ethanol and acetone demonstrate the potential of the sensor for use in high performance volatile organic compound (VOC) sensors. Owing to the low working temperature $\left(\sim 140^{\circ} \mathrm{C}\right)$, the nanocolumnar sensor shows very stable operation as illustrated in Fig. 3f. From a comparison involving the same configuration of chemical sensors under external heating (Supplementary Fig. S8), the working temperature of the present sensor is estimated to be lower than $150^{\circ} \mathrm{C}$. Meanwhile, the temperature of the backside of the sensor was measured to be $43^{\circ} \mathrm{C}$ during operation, at an applied bias voltage of $5 \mathrm{~V}$, confirming that the present sensor design undergoes minimal heat dissipation through the substrate. With increasing the applied bias voltage, we could obtain higher responses to target gases. However, when the applied bias voltage is higher than $7 \mathrm{~V}$, the working temperature of the sensor becomes higher than $200^{\circ} \mathrm{C}$. As we discussed earlier, such a high working temperature should come at a cost to long-term reliability probably along with morphological changes in the sensing film. This suggests that the applied bias voltage bias should be adjusted so that the working temperature may not exceed $200^{\circ} \mathrm{C}$.

In addition to high sensitivity and long-term reliability, low power consumption is required for practical applications of the nanocolumnar sensors as a component in handheld devices such as mobile phones. To address this issue, we have measured power consumption of the sensors with changing the area of sensing film and the duty cycle of pulsed bias voltage. Figure 4 a shows the power consumption of nanocolumnar sensors as a function of sensing area. By reducing the area of the $\mathrm{WO}_{3}$ sensing film from $1 \mathrm{~mm} \times 1 \mathrm{~mm}$ to $100 \mu \mathrm{m} \times$ $170 \mu \mathrm{m}$ (Supplementary Fig. S9), the response of the sensors remains nearly constant, while the power consumption decreases from 251 $\mu \mathrm{W}$ to $21.6 \mu \mathrm{W}$. These values are much lower than the power 
consumption of even micromachined thin film sensors (5-200 $\mathrm{mW})^{16,34}$ and comparable to those of self-heated single nanowire sensors (tens of $\mu \mathrm{W})^{17}$. When the response of the sensor with 100 $\mu \mathrm{m} \times 170 \mu \mathrm{m}$ sensing area is normalized with respect to the power consumption, it exhibits incomparably superior performance to the state-of-art chemoresistive sensors (Supplementary Fig. S10). The power consumption of the sensor could be further lowered using pulsed mode operation, as shown in Fig. $4 \mathrm{~b}$. When the duty cycle of the pulsed bias voltage is reduced down to $4 \%$, the sensor still exhibits excellent sensing performance with moderate decreases in response and response speed (Supplementary Fig. S11). The strikingly low power consumption, $0.18 \mu \mathrm{W}$, of the sensor at a $4 \%$ duty cycle means that the sensors can operate for a half year using a commercially available lithium polymer cell phone battery (output voltage: $3.7 \mathrm{~V} \mathrm{DC}$, capacity $1500 \mathrm{mAh}$ ). These results demonstrate the overall superior performance of the nanocolumnar $\mathrm{WO}_{3}$ thin film sensors and their remarkably low power requirements point to the feasibility of embedding them into miniature, portable devices.

In conclusion, we have realized all oxide transparent chemoresistive sensors with ultrahigh sensitivity, extremely low power consumption and excellent long-term stability by utilization of uniquely nanostructured metal oxide thin films, which are readily
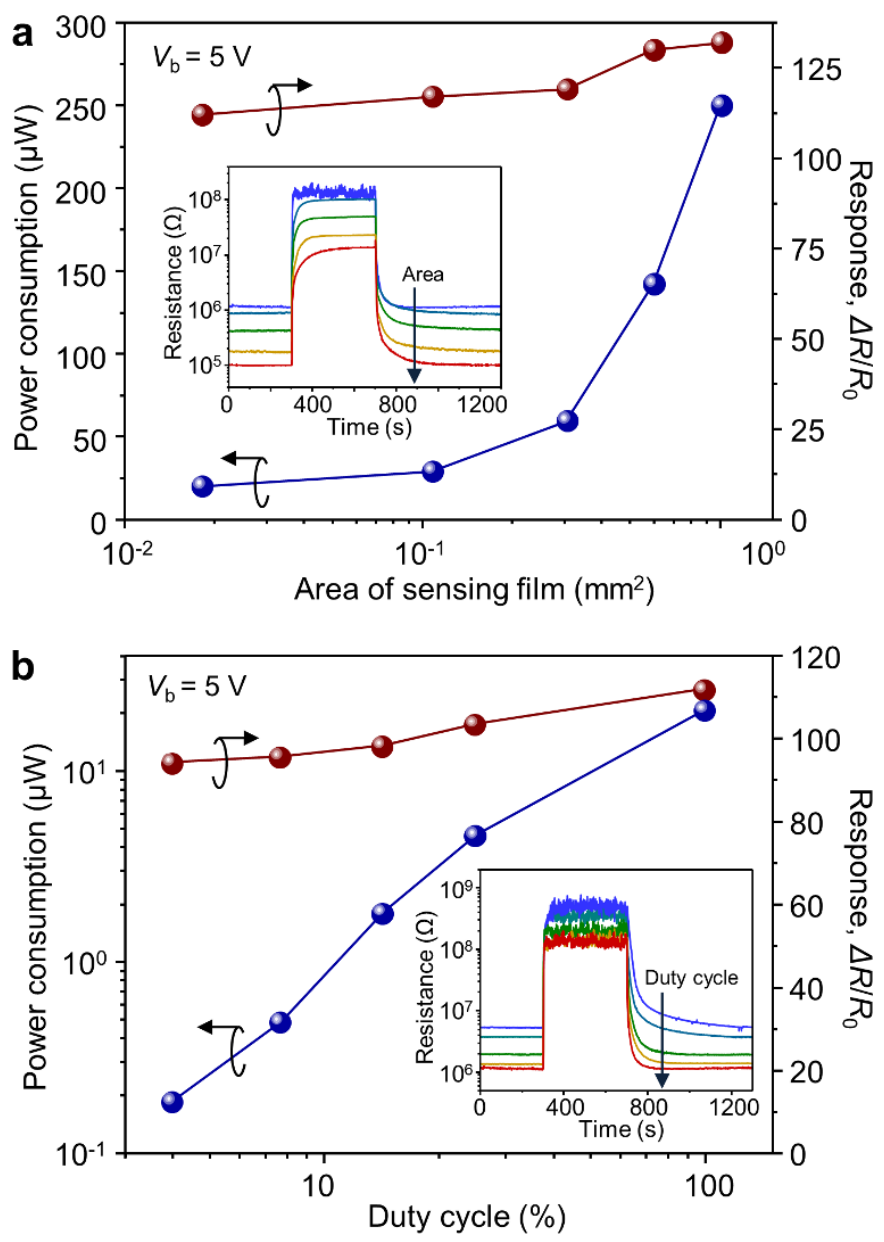

Figure 4| Power consumption. (a) Power consumption and response of nanocolumnar $\mathrm{WO}_{3}$ thin film sensors to $1 \mathrm{ppm} \mathrm{NO}_{2}$ as a function of the area of sensing film. Inset: response transients to $1 \mathrm{ppm} \mathrm{NO}_{2}$ of sensors with different areas of sensing film. (b) Power consumption and response of the nanocolumnar $\mathrm{WO}_{3}$ thin film sensor with $100 \mu \mathrm{m} \times 170 \mu \mathrm{m}$ sensing area to $1 \mathrm{ppm} \mathrm{NO}_{2}$ as a function of the duty cycle of pulsed bias voltage. For each duty cycle, the pulse period is $5 \mathrm{~ms}$. Inset: response transients to $1 \mathrm{ppm}$ $\mathrm{NO}_{2}$ of the sensor with changing the duty cycle. self-activated with $\mu \mathrm{A}$ current and 0.1 's of $\mu \mathrm{W}$ power levels. The remarkable device performance, achieved with a facile fabrication process, considerably broadens the potential application of chemoresistive sensors to transparent electronics and highly miniaturized mobile devices. We believe that very high performance portable electronic noses with selective chemical sensing can be developed by integrating multiple sensors of our design onto a single sensor platform coupled with the adoption of surface decoration ${ }^{35,36}$ and temperature variation ${ }^{37}$ techniques. The approach, successfully demonstrated in this work for miniaturized chemoresistive gas sensors, can be extended, we believe, to improve the performance of other solid-state devices such as fuel cells, $\mathrm{CO}_{2}$ reduction systems and other types of sensors that operate at elevated temperatures by removing separate heating elements and exploiting the selfactivation capacity of active elements within the device structure.

\section{Methods}

Sensor fabrication. ITO (150 nm thick, $10 \Omega / \square)$ IDE patterns of $5 \mu \mathrm{m}$ spacing were fabricated on glass substrates using photolithography and dry etching. After patterning the ITO IDEs, a $330 \mathrm{~nm}$ thick $\mathrm{WO}_{3}$ film was deposited onto predefined regions by a shadow mask on the ITO-IDE-patterned glass substrate using roomtemperature RF sputtering, where a polycrystalline $\mathrm{WO}_{3}$ target was utilized for film deposition. To render the film porous, the sputtering deposition was carried out at a glancing angle of $5^{\circ}$. The base pressure, working pressure, RF power, and gas flow rate were $2 \times 10^{-6} \mathrm{mTorr}, 3 \mathrm{mTorr}, 300 \mathrm{~W}$ and $30 \mathrm{sccm}$, respectively. The deposition rate of the film was $8 \mathrm{~nm} / \mathrm{min}$. The fabricated $\mathrm{WO}_{3} / \mathrm{ITO} /$ glass sensor structure was annealed in air at $500^{\circ} \mathrm{C}$ for $60 \mathrm{~min}$ to crystallize the amorphous $\mathrm{WO}_{3}$ film. For comparison, a planar $\mathrm{WO}_{3}$ thin film sensor was fabricated using on-axis $\mathrm{RF}$ sputtering, which leads to the deposition of a $330 \mathrm{~nm}$ thick dense $\mathrm{WO}_{3}$ film.

Characterization. The morphologies of the fabricated sensors were characterized by SEM (XL30 FEG ESEM, FEI) using $15 \mathrm{kV}$ voltage. TEM images were taken by a JEOL JEM $2100 \mathrm{~F}$ with a probe-Cs corrector. Cross-sectional TEM specimens of $\mathrm{WO}_{3}$ nanocolumns on a glass substrate were prepared by mechanical polishing followed by ion milling with Ar ions. The crystallinity and phase of the sensor films were characterized by glancing angle X-ray diffraction (D/Max-2500, Rigaku), where $\mathrm{Cu}$ $\mathrm{K} \alpha$ radiation (wavelength $=1.5418 \AA$ ) was used for the $\mathrm{X}$-ray source and the incident angle was fixed at a small angle $\left(2^{\circ}\right)$. For all the $\mathrm{WO}_{3}$ sensing films, selected area electron diffraction patterns from TEM and X-ray diffraction patterns could be indexed with the pure monoclinic phase of $\mathrm{WO}_{3}$ with lattice constants $a=5.261 \AA$, $b=5.128 \AA, c=7.650 \AA$ and $\beta=92.05^{\circ}$ (JCPDS \#88-0550). Thermographic images of the sensors were obtained with an infrared camera (SC5000, FLIR). The transmittance of the entire sensor structures was measured over the wavelength range of 200-900 nm by UV/VIS spectrometry (Lambda 18, Perkin Elmer). NanoECR measurements were carried out using a nanomechanical test instrument (TI $750 \mathrm{Ubi}$, Hysitron).

Sensor measurements. The gas sensing properties of the fabricated thin film sensors were measured without external heating. As the flow gas was changed from dry air to a calibrated test gas (balanced with dry air, Sinyang Gases), the variation in sensor resistance was monitored using a source measurement unit (Keithley 236). A constant flow rate of $500 \mathrm{sccm}$ was used for dry air and the test gas. The sensor resistance was measured under a DC bias voltage of $0.1-5 \mathrm{~V}$. The response of the sensors $\left(\Delta R / R_{0}\right.$ for $\mathrm{NO}_{2}, \Delta I / I_{0}$ for ethanol, acetone, $\mathrm{SO}_{2}, \mathrm{NH}_{3}, \mathrm{H}_{2}$, and $\mathrm{CO}$ ) was accurately determined by measuring the baseline resistances of the sensors in dry air and the fully saturated resistances after exposure to the test gas. Gas flow was controlled using mass flow controllers, and all measurements were recorded to a computer through the use of LabVIEW over a GPIB interface. The current-voltage characteristics of the fabricated sensors were measured to check the contribution of the contact resistance between ITO IDEs and the $\mathrm{WO}_{3}$ sensing film to the overall performance. For the pulse mode operation, the pulse period of $5 \mathrm{~ms}$ was used and the pulse duration was changed from $1.25 \mathrm{~ms}$ ( $25 \%$ duty cycle) to $0.2 \mathrm{~ms}$ ( $4 \%$ duty cycle). Additional experimental details about sensor measurements may be found in our previous reports ${ }^{38,39}$.

1. Cui, Y., Wei, Q. Q., Park, H. K. \& Lieber, C. M. Nanowire nanosensors for highly sensitive and selective detection of biological and chemical species. Science 293, 1289-1292 (2001).

2. Star, A., Joshi, V., Skarupo, S., Thomas, D. \& Gabriel, J. C. P. Gas sensor array based on metal-decorated carbon nanotubes. J. Phys. Chem. B 110, 21014-21020 (2006).

3. Sysoev, V. V., Button, B. K., Wepsiec, K., Dmitriev, S. \& Kolmakov, A. Toward the nanoscopic "electronic nose": Hydrogen vs carbon monoxide discrimination with an array of individual metal oxide nano- and mesowire sensors. Nano Lett. 6, 1584-1588(2006).

4. McAlpine, M. C., Ahmad, H., Wang, D. W. \& Heath, J. R. Highly ordered nanowire arrays on plastic substrates for ultrasensitive flexible chemical sensors. Nat. Mater. 6, 379-384 (2007). 
5. Tricoli, A. \& Pratsinis, S. E. Dispersed nanoelectrode devices. Nat. Nanotechnol. 5, $54-60$ (2010).

6. Sysoev, V. V. et al. Single-nanobelt electronic nose: engineering and tests of the simplest analytical element. ACS Nano 4, 4487-4494 (2010).

7. Lu, Y. J., Meyyappan, M. \& Li, J. Trace detection of hydrogen peroxide vapor using a carbon-nanotube-based chemical sensor. Small 7, 1714-1718 (2011).

8. Park, J. U., Nam, S. U., Lee, M. S. \& Lieber, C. M. Synthesis of monolithic graphene-graphite integrated electronics. Nat. Mater. 11, 120-125 (2012).

9. Chen, G. G., Paronyan, T. M., Pigos, E. M. \& Harutyunyan, A. R. Enhanced gas sensing in pristine carbon nanotubes under continuous ultraviolet light illumination. Sci Rep 2, 343 (2012)

10. Capone, S. et al. Solid state gas sensors: State of the art and future activities. J. Optoelectron. Adv. Mater. 5, 1335-1348 (2003).

11. Eranna, G., Joshi, B. C., Runthala, D. P. \& Gupta, R. P. Oxide materials for development of integrated gas sensors - A comprehensive review. Crit. Rev. Solid State Mater. Sci. 29, 111-188 (2004).

12. Barsan, N., Koziej, D. \& Weimar, U. Metal oxide-based gas sensor research: How to? Sens. Actuators B 121, 18-35 (2007).

13. Yamazoe, N. \& Shimanoe, K. New perspectives of gas sensor technology. Sens. Actuators B 138, 100-107 (2009).

14. Lee, J. H. Gas sensors using hierarchical and hollow oxide nanostructures: Overview. Sens. Actuators B 140, 319-336 (2009).

15. Comini, E. et al. Quasi-one dimensional metal oxide semiconductors: Preparation, characterization and application as chemical sensors. Prog. Mater. Sci. 54, 1-67 (2009)

16. Simon, T., Barsan, N., Bauer, M. \& Weimar, U. Micromachined metal oxide gas sensors: opportunities to improve sensor performance. Sens. Actuators B 73, $1-26$ (2001).

17. Prades, J. D. et al. Ultralow power consumption gas sensors based on self-heated individual nanowires. Appl. Phys. Lett. 93, 123110 (2008).

18. Strelcov, E. et al. Evidence of the self-heating effect on surface reactivity and gas sensing of metal oxide nanowire chemiresistors. Nanotechnology 19, 355502 (2008).

19. Choi, K. J. \& Jang, H. W. One-Dimensional Oxide Nanostructures as Gas-Sensing Materials: Review and Issues. Sensors 10, 4083-4099 (2010).

20. Hernandez-Ramirez, F. et al. On the role of individual metal oxide nanowires in the scaling down of chemical sensors. Phys. Chem. Chem. Phys. 11, 7105-7110 (2009).

21. Sysoev, V. V. et al. Percolating $\mathrm{SnO}_{2}$ nanowire network as a stable gas sensor: Direct comparison of long-term performance versus $\mathrm{SnO}_{2}$ nanoparticle films. Sens. Actuators B 139, 699-703 (2009).

22. Rothschild, A. \& Komem, Y. On the relationship between the grain size and gassensitivity of chemo-resistive metal-oxide gas sensors with nanosized grains. J. Electroceram. 13, 697-701 (2004).

23. Korotcenkov, G. et al. Structural stability of indium oxide films deposited by spray pyrolysis during thermal annealing. Thin Solid Films 479, 38-51 (2005).

24. Xu, C. N., Tamaki, J., Miura, N. \& Yamazoe, N. Grain-size effects on gas sensitivity of porous $\mathrm{SnO}_{2}$-based elements. Sens. Actuators B 3, 147-155 (1991).

25. Pinna, N., Neri, G., Antonietti, M. \& Niederberger, M. Nonaqueous synthesis of nanocrystalline semiconducting metal oxides for gas sensing. Angew. Chem. Int. Ed. 43, 4345-4349 (2004).

26. Franke, M. E., Koplin, T. J. \& Simon, U. Metal and metal oxide nanoparticles in chemiresistors: Does the nanoscale matter? Small 2, 36-50 (2006).

27. Robbie, K. \& Brett, M. J. Sculptured thin films and glancing angle deposition: Growth mechanics and applications. J. Vac. Sci. Technol. A 15, 1460-1465 (1997).

28. Hawkeye, M. M. \& Brett, M. J. Glancing angle deposition: Fabrication, properties, and applications of micro- and nanostructured thin films. J. Vac. Sci. Technol. A 25, 1317-1335 (2007)

29. Xi, J. Q. et al. Optical thin-film materials with low refractive index for broadband elimination of Fresnel reflection. Nat. Photonics 1, 176-179 (2007).
30. Shim, Y. S. et al. Transparent conducting oxide electrodes for novel metal oxide gas sensors. Sens. Actuators B 160, 357-363 (2011).

31. Wager, J. F. Keszler, D. A. \& Presley, R. E. Transparent Electronics (Springer, New York, 2008).

32. Li, J. et al. Carbon nanotube sensors for gas and organic vapor detection. Nano Lett. 3, 929-933 (2003).

33. Dua, V. et al. All-organic vapor sensor using inkjet-printed reduced graphene oxide. Angew. Chem. Int. Ed. 49, 2154-2157 (2010).

34. Elmi, I., Zampolli, S., Cozzani, E., Mancarella, F. \& Cardinali, G. C. Development of ultra-low-power consumption $\mathrm{MO}_{\mathrm{X}}$ sensors with ppb-level VOC detection capabilities for emerging applications. Sens. Actuators B 135, 342-351 (2008).

35. Kolmakov, A., Klenov, D. O., Lilach, Y., Stemmer, S. \& Moskovits, M. Enhanced gas sensing by individual $\mathrm{SnO}_{2}$ nanowires and nanobelts functionalized with $\mathrm{Pd}$ catalyst particles. Nano Lett. 5, 667-673 (2005).

36. Baik, J. M. et al. Tin-oxide-nanowire-based electronic nose using heterogeneous catalysis as a functionalization strategy. ACS Nano 4, 3117-3122 (2010).

37. Sysoev, V. V., Goschnick, J., Schneider, T., Strelcov, E. \& Kolmakov, A. A gradient microarray electronic nose based on percolating $\mathrm{SnO}_{2}$ nanowire sensing elements. Nano Lett. 7, 3182-3188 (2007).

38. Moon, H. G. et al. Highly sensitive $\mathrm{CO}$ sensors based on cross-linked $\mathrm{TiO}_{2}$ hollow hemispheres. Sens. Actuators B 149, 116-121 (2010).

39. Moon, H. G. et al. Embossed $\mathrm{TiO}_{2}$ thin films with tailored links between hollow hemispheres: synthesis and gas-sensing properties. J. Phys. Chem. C 115, 9993-9999 (2011).

\section{Acknowledgments}

We thank W. I. Park for SEM measurements and appreciate S. H. Hong and K. T. Nam for fruitful discussions. The authors gratefully acknowledge support from the Korea Ministry of Intelligence and Economy (contract no. K0004114), the Korea Institute of Science and Technology (grant no. 2E22121) and a research program of the Korea Ministry of Environment. H.G.M. is supported by a Hi Seoul Science Fellowship from the Seoul Scholarship Foundation. H.W.J. is grateful to Seoul National University for financial support.

\section{Author contributions}

H.G.M. performed film deposition, characterizations and sensor measurements. Y.S.S. and D.H.K. fabricated the sensors. H.Y.J. and M.J. carried out TEM measurements. J.Y.J. and S.M.H. conducted NanoECR measurements. J.K.K., J.S.K., H.H.P., J.H.L, H.W.J. and S.J.Y supervised the experiments and contributed to manuscript preparation. H.G.M., J.K.K., J.H.L., H.W.J., S.J.Y. and H.L.T. analysed the data and wrote the manuscript. H.L.T. provided advice for the research. S.J.Y. and H.W.J. initiated and directed the research. H.W.J. conceived the idea and designed the experiments. All authors discussed the progress of research and reviewed the manuscript.

\section{Additional information}

Supplementary information accompanies this paper at http://www.nature.com/ scientificreports

Competing financial interests: The authors declare no competing financial interests.

License: This work is licensed under a Creative Commons

Attribution-NonCommercial-NoDerivative Works 3.0 Unported License. To view a copy of this license, visit http://creativecommons.org/licenses/by-nc-nd/3.0/

How to cite this article: Moon, H.G. et al. Self-activated ultrahigh chemosensitivity of oxide thin film nanostructures for transparent sensors. Sci. Rep. 2, 588; DOI:10.1038/srep00588 (2012). 